\title{
The DSM- 5 diagnosis of nonsuicidal self-injury disorder: a review of the empirical literature
}

\author{
Maria Zetterqvist ${ }^{1,2^{*}}$
}

\begin{abstract}
With the presentation of nonsuicidal self-injury disorder (NSSID) criteria in the fifth version of the Statistical and Diagnostic Manual of Mental Disorders (DSM-5), empirical studies have emerged where the criteria have been operationalized on samples of children, adolescents and young adults. Since NSSID is a condition in need of further study, empirical data are crucial at this stage in order to gather information on the suggested criteria concerning prevalence rates, characteristics, clinical correlates and potential independence of the disorder. A review was conducted based on published peer-reviewed empirical studies of the DSM-5 NSSID criteria up to May 16, 2015. When the DSM-5 criteria were operationalized on both clinical and community samples, a sample of individuals was identified that had more general psychopathology and impairment than clinical controls as well as those with NSSI not meeting criteria for NSSID. Across all studies interpersonal difficulties or negative state preceding NSSI was highly endorsed by participants, while the distress or impairment criterion tended to have a lower endorsement. Results showed preliminary support for a distinct and independent NSSID diagnosis, but additional empirical data are needed with direct and structured assessment of the final DSM-5 criteria in order to reliably assess and validate a potential diagnosis of NSSID.
\end{abstract}

Keywords: Nonsuicidal self-injury disorder, Adolescents, DSM-5, Review

\section{Background}

Nonsuicidal self-injury (NSSI), defined as the deliberate, self-inflicted destruction of body tissue without suicidal intent and for purposes not socially sanctioned, includes behaviors such as cutting, burning, biting and scratching skin [1]. NSSI is especially prevalent during adolescence with mean and pooled rates of $17-18 \%$ in recent reviews of community samples [2, 3]. In clinical samples of adolescents rates are even higher, with $40 \%$ or more reporting NSSI [4]. During the last decades there have been ongoing discussions regarding the conceptualization and diagnostic organization of NSSI. In the diagnostic nomenclature NSSI has been limited to a symptom of borderline personality disorder (BPD), described as suicidal behavior, gestures, threats or self-mutilating behavior [5].

\footnotetext{
*Correspondence: maria.zetterqvist@liu.se

${ }^{1}$ Department of Clinical and Experimental Medicine, Linköping

University, 58185 Linköping, Sweden

Full list of author information is available at the end of the article
}

Arguments have been put forward that NSSI should be a separate syndrome [6-11]. In the early 1980s Pattison and Kahan [11] and Kahan and Pattison [9] described the typical patterns of a separate deliberate self-harm syndrome, proposing that it should be included in the fourth version of the Diagnostic and Statistical Manual of Mental Disorders (DSM-IV) [5], with inability to resist the impulse to injure oneself, increased sense of tension prior to the act and experience of release/relief after the act as essential features. Later, Favazza and Rosenthal [6, 7] suggested DSM inclusion of a repetitive self-mutilation syndrome and complemented earlier descriptions by adding preoccupation with harming oneself. In 2005 Muehlenkamp [10] also proposed that self-injurious behavior should be a separate clinical syndrome, emphasizing the absence of conscious suicidal intent, the inability to resist NSSI impulses, the negative affective/cognitive state prior to and the relief after NSSI, as well as the preoccupation with and repetitiveness of the behavior. These earlier features overlap to a large extent with the suggested Shaffer and 
Jacobson [12] NSSI criteria proposed to the DSM-5 [13] Childhood Disorder and Mood Disorders work group for inclusion as a DSM-5 disorder, in that they describe the functional, motivational and emotional aspects of NSSI [14]. The criteria have been revised several times during the work progress, mainly concerning their organization $[12,13,15]$.

Shaffer and Jacobson [12] pinpointed several reasons in their rationale for reclassifying NSSI: NSSI is associated with clinical and functional impairment; the classification of NSSI solely as a symptom of BPD is inconsistent with recent evidence; NSSI needs to be separated from suicide attempts; studying NSSI purely within a BPD context or as a manifestation of suicidality will hamper research and treatment of NSSI; a standardized definition of clinically significant NSSI would facilitate comparisons of findings from different studies and improve communication and clarity in clinical care.

There is general consensus that there is an association between BPD and NSSI [16-19], but that NSSI is not unique to BPD. NSSI is also associated with other personality disorders $[19,20]$ and to several axis I symptomatologies [16, 19-21], and may also be present without any psychiatric comorbidities [22]. To classify NSSI purely as a criterion of BPD implies that it does not have clinical significance outside the BPD context [23].

Furthermore, not separating suicidal behaviors and NSSI can lead to inaccurate case conceptualization, risk assessment, treatment and iatrogenic hospitalization [23]. Empirical differences have been found between adolescents engaging in different kinds of self-injurious behaviors with and without suicidal intent (e.g., [18]). Ignoring intent in describing self-injury can lead to an overestimation of the prevalence of suicide attempts and prevent correct identification of specific risk factors for the respective behaviors [24]. The relationship between NSSI and suicide attempts is complex and nuanced [25] and there is general agreement that there is an overlap between nonsuicidal and suicidal self-injury [20, 26]. Recent longitudinal research has found that NSSI predicts suicide attempts in adolescents [27-29] and that the high co-occurrence between the two can be understood in the light of NSSI increasing the risk for suicidal behavior [30]. Arguments have thus been put forward that nonsuicidal and suicidal self-injury need to be differentiated on the basis of differences in intent, lethality, methods, prevalence, frequency and functions [10,31]. It has also been argued that new definitions of NSSI disorder and suicidal behavior disorder would facilitate comparisons between studies [32].

Despite the fact that NSSI is prevalent and impairing in adolescents, it has not been given any psychopathological significance except as a symptom of BPD until DSM-5
[22]. Improved communication, more precise definition and clearer implications for prognosis and treatment are thus advocated [22, 33], allowing NSSI to be highlighted and treated outside the BPD context [22, 34, 35]. However, doubts have also been voiced [36], mainly concerning the issue of suicidal intent and how the relationship between NSSI and suicidal behaviors should be conceptualized. Critics argue that suicidal or nonsuicidal intent is wrongly reduced to a dichotomy, instead of being conceptualized as a multidimensional construct where the ambiguity and the difficulty in arriving at a valid and reliable assessment of intent need to be acknowledged. Critics further claim that the term nonsuicidal is questionable due to the aforementioned overlap between suicidal thoughts and behaviors and NSSI. There is also concern that a diagnosis could increase stigmatization in a young age group and that the lack of empirical support for an NSSI diagnosis argues for caution at this stage $[37,38]$.

Due to the novelty of the suggested NSSI criteria, crucial empirical data have only recently begun to emerge [39]. The NSSI criteria were finally placed in Section III of DSM-5: Emerging Measures and Models, as a condition that requires further study [13], due to lack of reliability in the clinical trial. Two of the child/adolescent sites had inadequate sample sizes, which were insufficient to obtain accurate estimates of kappa. The third field trial was successful, but the test-retest reliability was unacceptable $[40,41]$. Since empirical data are crucial at this point of the diagnostic process, this paper aims at reviewing the empirical literature on the NSSI disorder (NSSID) diagnosis up to the present time.

\section{Method}

Electronic searches were made using the scholarly database search engines Pubmed, PsycInfo, Scopus and Academic Search Premier up to May 16, 2015. The following search terms were used: "non-suicidal self-injury" AND "dsm"; "nonsuicidal self-injury" AND "dsm"; "self-injury" AND "dsm"; "self-harm" AND "dsm". Abstracts of identified articles were reviewed for inclusion and exclusion criteria. In addition, reference lists of articles were checked so as not to miss other articles that had not appeared in the electronic search. Articles were included if they were peer-reviewed empirical research of the suggested DSM-5 NSSI criteria on samples with children, adolescents and young adults and were written in English. Since empirical data on the NSSI diagnosis are only now emerging, the few articles concerning adults only were also included, but presented separately.

\section{Results}

A total of 16 published studies were found that presented empirical data on NSSID. Four studies used the final 
DSM-5 [13] criteria, while others used some or all of the earlier criteria $[12,15]$. Of these, one based the empirical data on clinicians' ratings [42] and two [43, 44] were new analyses of study populations already included [45, 46]. Ten studies included adolescents [14, 23, 44, 46-52], of which two also included older children [47, 48]. Four studies included young adults [51-54] (only or in addition to adolescents) and three were limited to adults only $[43,45,55]$. See Table 1 for empirical studies.

\section{NSSI disorder characteristics}

Prevalence of NSSID in child and adolescent community samples ranged from 1.5 to $5.6 \%$ [47, 48]. In community samples of adolescents only, $3.1-6.7 \%$ met NSSID criteria $[14,46]$, as compared to $18.8 \%$ of those with an NSSI history [46] and $49.2 \%$ of those with repetitive NSSI [14]. Equivalent rates in a young adult community sample with repetitive NSSI were 37\% [53]. Prevalence in adolescent and young adult clinical samples ranged from 36.9 to $50 \%$ $[23,49]$ while 46.2 to $78 \%$ [23, 50-52] of those with an NSSI history met NSSID criteria. In most studies more girls than boys met criteria (Table 1). The average age of onset for NSSI in those with NSSID ranged from 12.52 to 13.05 years (SD 1.73-3.53) [23, 50, 52]. The most common methods were cutting, banging/hitting, severe scratching, carving and scraping $[23,50,53]$. Several methods were reported, ranging from an average of 4.29-8 (SD 2.18-2.78) methods [23, 46, 50-53]. The functions most often endorsed by those who met NSSID criteria were affect regulation, self-punishment and anti-dissociation/feeling-generation [23, 46, 50,53]. In clinical studies of adolescents and young adults with NSSID, 69.2-83.3\% [50, 51] reported having made a suicide attempt, and in one study $24.4 \%$ reported having done so during the last month [23]. Among community adolescents who met criteria for NSSID, $20 \%$ reported that at least one of their self-injuries during the last year was a suicide attempt [46]. Several of those with NSSID in clinical and community samples with recurrent NSSI also had concurrent axis I diagnoses [23, 45, 50, 51, 53]. Mood disorders commonly co-occurred, with examples of 72.5\% [53] and 79.5\% [50] for depression. Anxiety disorders were also commonly reported (72.5-89\%) [23, 51, 53], as was posttraumatic stress disorder (PTSD) with rates of $25.0-28.2 \%$ [50, 53]. In two studies of clinical adolescents with NSSID, 51.7\% [23] and 20.5\% [50] met criteria for BPD. High levels of emotional dysregulation [23, 53], low quality of life [52] and impairment [45, 52] have also been found in those meeting criteria for NSSID.

\section{DSM-5 NSSI criteria Criterion A}

In a self-injuring sample of inpatient and intensive outpatient adolescents and young adults, $85.5 \%$ endorsed criterion A, i.e., at least 5 days [52]. Rates of $76-77 \%$ were found in an outpatient clinical sample and also in a community sample of repetitive NSSI [51, 53], whilst a considerably lower endorsement of criterion A (20.8\%) was found in a self-injuring adult community sample [55]. Of those who met NSSID criteria, 73.7\% had performed NSSI $\geq 11$ times during the last year and $26.3 \%$ had done so 5-10 times. More girls than boys had performed NSSI $\geq$ five times in this study of community adolescents [46]. Lengel and Mullins-Sweatt [42] asked 119 clinicians and NSSI experts to rate whether the NSSID criteria represented prototypic cases/symptoms of a self-injuring patient and $85 \%$ considered that five instances was prototypic. Absence of suicidal intent was endorsed as prototypic by $90 \%$.

\section{Criterion B}

In one community study of adolescents [46], almost all (99.5\%) of those with NSSID reported having engaged in NSSI with the expectation of relieving an interpersonal difficulty or negative feeling, or of inducing a positive feeling. A similarly high endorsement (87.2-87.7\%) was found in inpatient adolescents with NSSID [50, 52]. Engaging in NSSI for a purpose was also thought to be a prototypical symptom by $71.9 \%$ of clinicians and NSSI experts [42]. In one study [53] 79\% of young adults with NSSI met criterion B, compared to $66.4 \%$ in an adult community sample of self-injurers [55]. The earlier B criterion (current DSM-5 equivalent of B and C) was met by $97 \%$ of self-injuring outpatient adolescents and young adults [51]. Empirical studies that used the final DSM-5 [13] criteria and presented data for each subcriterion found B1 (relief) to be the most common [52, 55]. In adolescents, B3 (positive feeling) was least commonly endorsed [52]. Criterion B2 (to relieve interpersonal problems) was more often endorsed in a clinical sample including adolescents [52] than in an adult community sample [55]. In the study by Washburn and colleagues [52] patients rarely met criterion $B$ without also meeting criterion C. Criterion B was further found to be associated with interpersonal functions of NSSI [53]. Girls reported expectations of relief from negative feelings and thoughts more often than boys [47].

\section{Criterion C}

Criterion C1 (interpersonal/psychological precipitant) was consistently met by nearly all participants. Of adolescents with NSSID, 97.4-100\% endorsed criterion C1 $[46,50,52]$. In the study by Washburn and colleagues [52] there was an additionally high endorsement of criteria C2 (preoccupation) and C3 (urge). Of those who did not meet criteria for NSSID, very few failed to meet criterion C. Criterion C1 was also significantly associated 


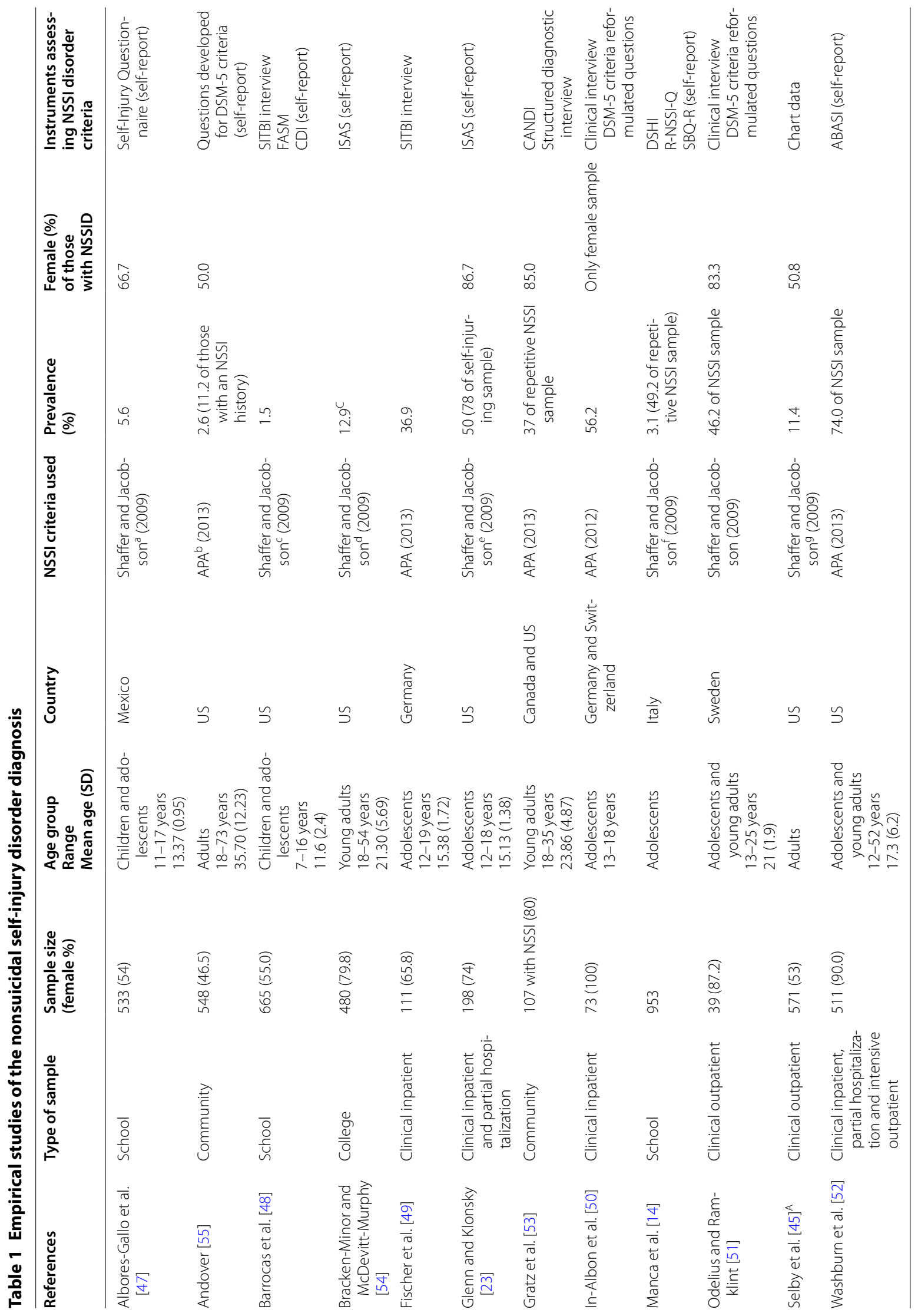




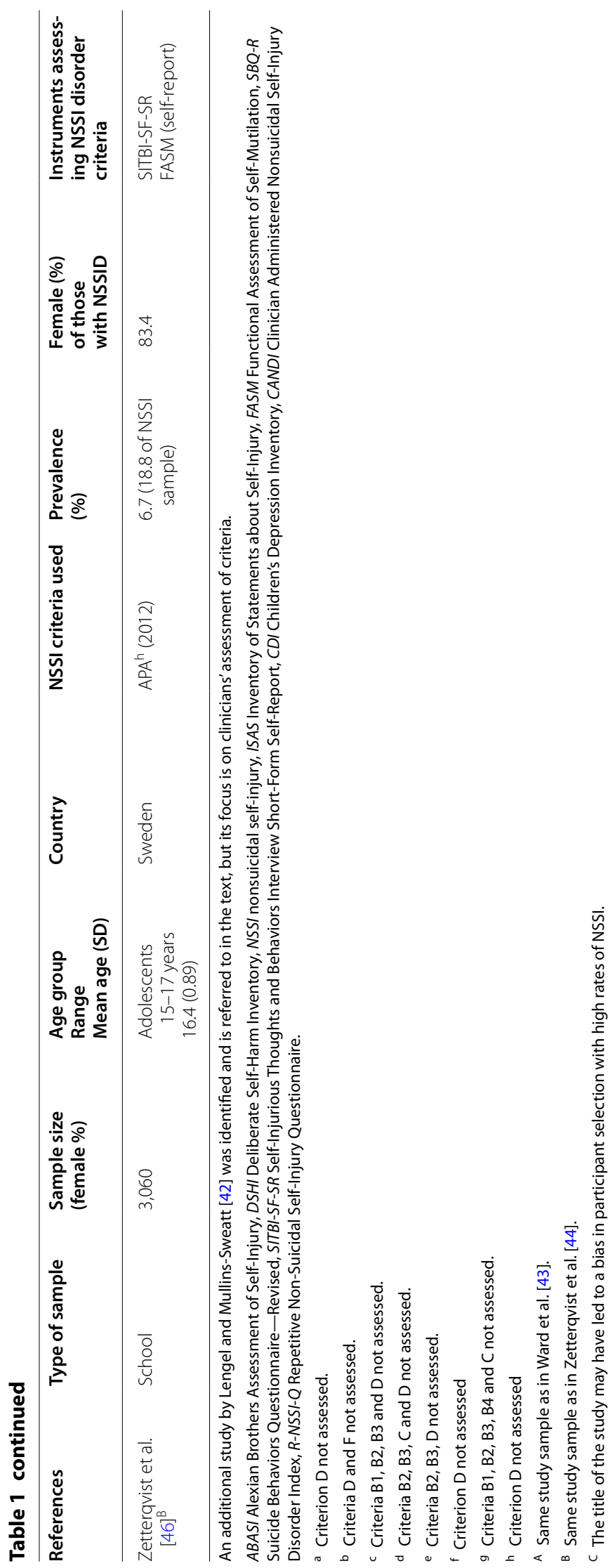


with psychopathology and impairment [52]. Of those with self-injury, 81-98\% [23, 51-53] met criterion $C$ and $82.4 \%$ of self-injuring community adults met criterion $\mathrm{C} 1$ [55]. Psychological precipitants were more commonly reported in girls [46, 47]. Negative emotions/thoughts prior to NSSI was considered a prototypic symptom by $87.5 \%$ of clinicians, while frequent urge and preoccupation to engage in NSSI was relatively less so [42]. Similarly, preoccupation was reported by less than $50 \%$ of the adolescents with NSSID in the study by In-Albon and colleagues [50], while frequent urge was endorsed by $89.7 \%$.

\section{Criterion D}

In a study of young adults [53] 91\% of self-injurers met criterion $\mathrm{D}$, which refers to behaviors that are not socially sanctioned. Eighty-eight percent of clinicians and NSSI experts thought this to be a prototypic symptom [42].

\section{Criterion $E$}

In one study of clinical self-injuring adolescents and young adults, $43 \%$ failed to meet NSSID criteria because they did not fulfill the distress or interference criterion [51]. The interviewers considered this criterion difficult to assess, since patients tended to report that their selfharm was helpful rather than distressing or impairing. In self-injuring samples, $41-64 \%$ met criterion $E[51,53]$. In adolescents with NSSID, 76.8\% [46] and 69.2\% [50] reported that their NSSI caused them distress. However, a question whether adolescents desired help for their NSSI received a 79.5\% endorsement [50]. In Andover's [55] adult sample, $8.8 \%$ of self-injurers endorsed interferences in functioning, while $60.8 \%$ wanted to stop engaging in NSSI. The most common interferences reported were in academic and social (school) life [47], interpersonal relationships and schooling [46] and also leisure time [50]. More girls than boys acknowledged distress/ impairment [46]. Criterion $\mathrm{E}$ had less than $50 \%$ endorsement as a prototypic symptom [42]. In a study of young adults, clinical characteristics such as emotion dysregulation, BPD, symptoms of depression, anxiety and stress were most strongly associated with criterion E, as were intrapersonal functions, and this criterion best distinguished those with NSSID from those with NSSI without NSSID [53].

\section{Criterion $F$}

In a self-injuring sample of young adults, $80 \%$ met exclusion criterion F [53], as did $98.2 \%$ of adolescents [52]. Several of the studies using self-report measures did not assess this criterion directly.

\section{NSSI disorder versus NSSI, clinical controls and borderline personality disorder NSSI disorder versus NSSI}

Compared to those with NSSI not meeting NSSID criteria, those with NSSID reported higher levels of psychopathology and significantly more interference in functioning $[52,53,55]$, as well as more variety of NSSI methods [51-53] (Table 2). The NSSID group endorsed significantly higher levels of automatic functions (emotion relief, feeling generation) than the non-NSSID group $[46,53,55]$, with average rates of automatic negative reinforcement of $2.43(0.84)$ vs. $1.54(0.81)$ and automatic positive reinforcement $2.08(0.71)$ vs. $1.33(0.51)$ in inpatient adolescents [50]; significantly higher levels of emotion dysregulation, 109.42 (21.79) vs. 94.26 (23.07) [53]; significantly higher levels of symptoms of depression, 18.68 (11.28) vs. 13.99 (9.86) indicating moderate vs. mild/moderate symptoms; anxiety symptoms, 15.12 (9.81) vs. 9.31 (7.23) indicating severe vs. mild symptoms and stress, 20.65 (10.00) vs. 14.20 (8.04) indicating moderate vs. mild symptoms in young adults with recurrent NSSI [53]. There were also significantly higher levels of symptoms of depression, anxiety, anger, posttraumatic stress and dissociation in community adolescents with NSSID compared to those with NSSI not meeting NSSID criteria [44] and significantly more smoking and drug use [46]. Significantly more community adolescents with NSSID reported experiences of adversities and maltreatment than adolescents with NSSI not meeting NSSID criteria [44], for example, bullying, 62.4 vs. $40.0 \%$; emotional abuse, 77.4 vs. $40.8 \%$; physical abuse from an adult within the family, 38.7 vs. $16.0 \%$ and sexual abuse, 36.6 vs. 8.4\% [44]. Suicide ideation, 1.40 (1.17) vs. 1.08 (1.18), was also significantly higher in inpatient adolescents with NSSID compared to those with NSSI not meeting full criteria [52]. Concerning concurrent axis I diagnoses, significantly more young adults with NSSID had PTSD, 25.0 vs. $10.4 \%$; $\mathrm{BPD}, 45.0$ vs. $19.4 \%$; bipolar disorder, 20.0 vs. 6.0\%; social anxiety disorder, 37.5 vs. $19.4 \%$ and alcohol dependence, 40.0 vs. $17.9 \%$, compared to individuals with recurrent NSSI not meeting NSSID criteria [53]. Among inpatient adolescents with NSSID there were significantly higher levels of BPD traits, 37.79 (11.35) vs. 33.38 (10.92) [52]. Importantly, the association between NSSID and psychopathology in the study by Gratz and colleagues [53] remained significant when controlling for BPD.

\section{NSSI disorder versus clinical controls}

Significantly more inpatient adolescents with NSSID reported suicide ideation, 67.1 vs. $29.2 \%$ and suicide attempts, 24.4 vs. $8.6 \%$ [23], compared to clinical adolescents. Furthermore, significantly more inpatient 


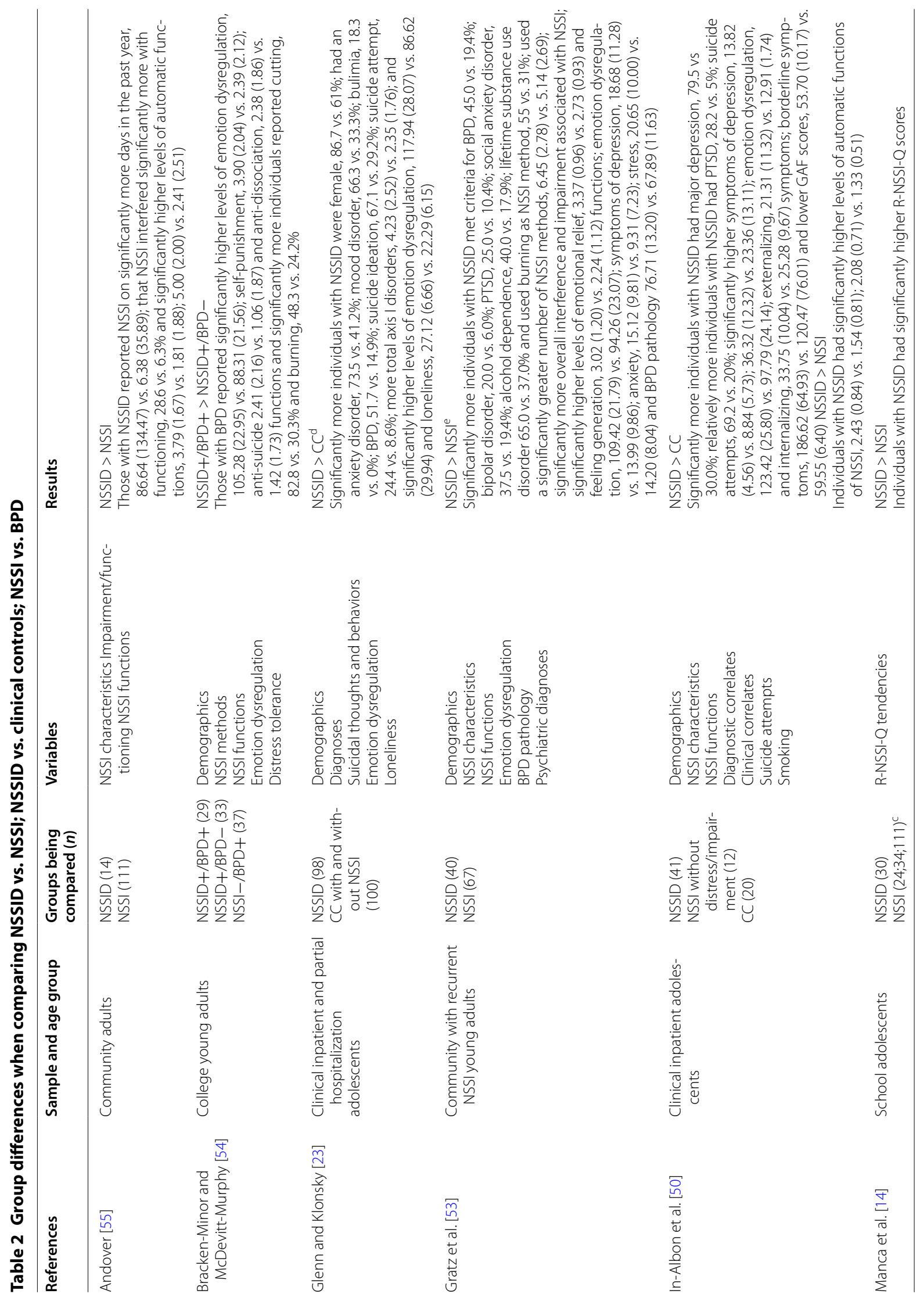




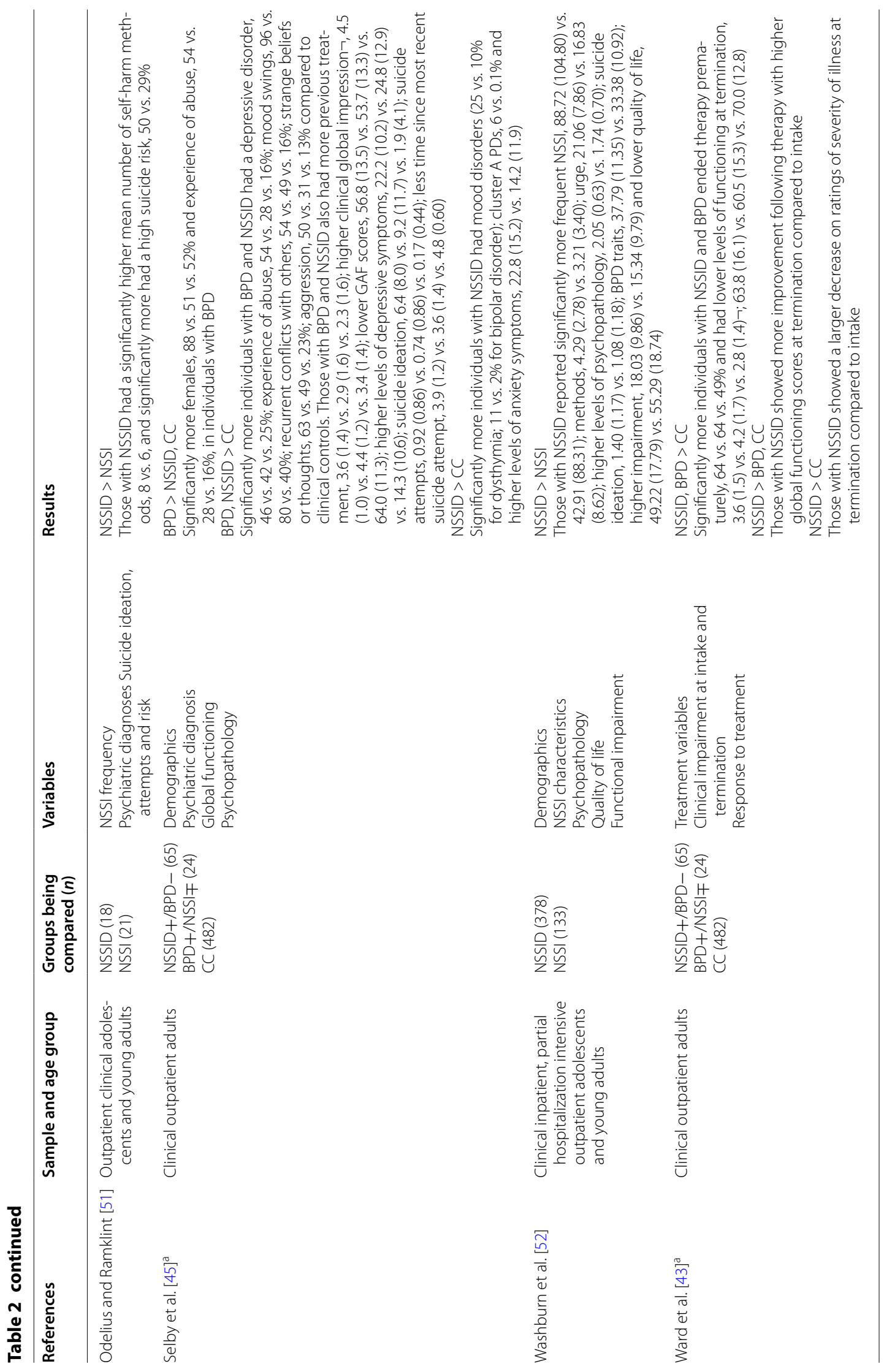




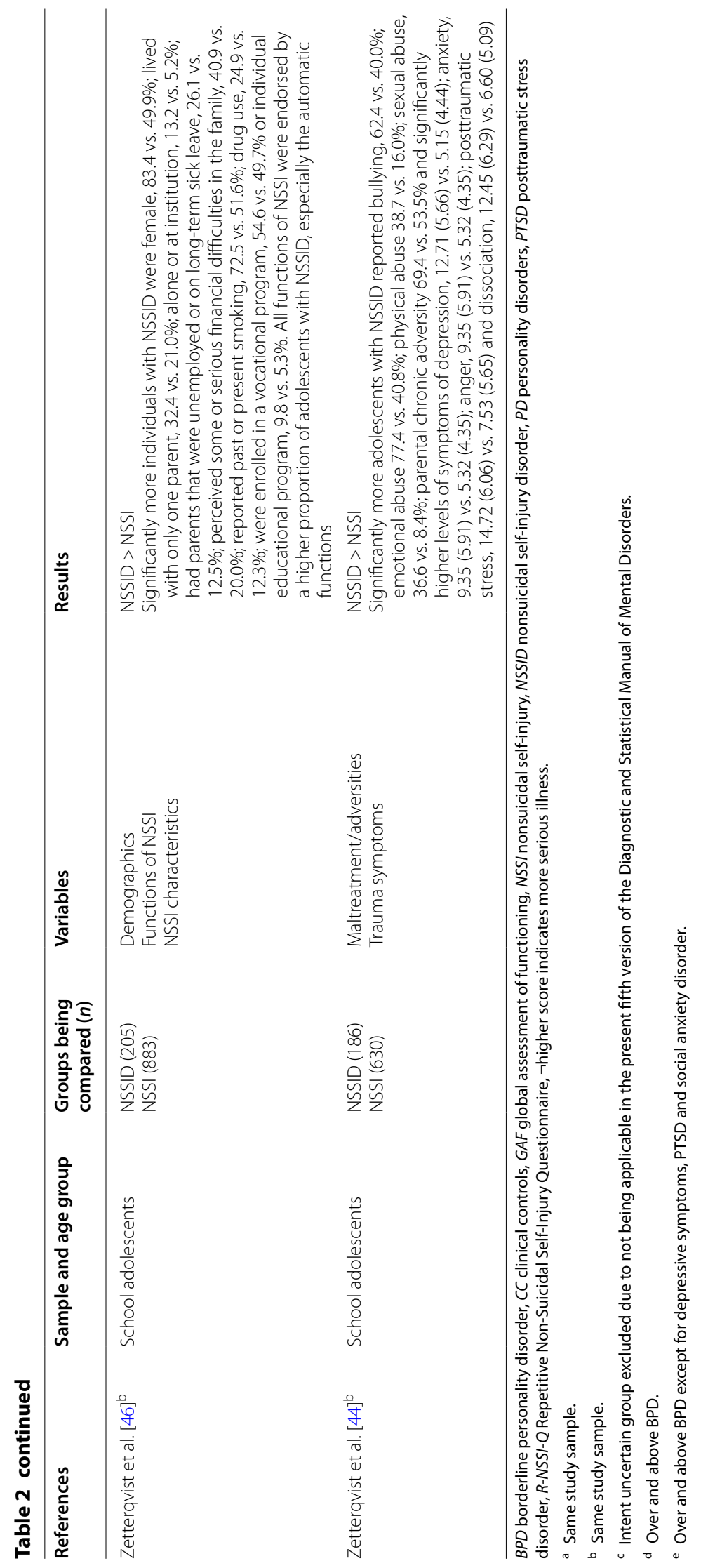


adolescents among those who met NSSID criteria had major depression, 79.5 vs. $30.0 \%$ [50]; anxiety disorder, 73.5 vs. $41.2 \%$; mood disorder, 66.3 vs. $33.3 \%$; bulimia, 18.3 vs. $0 \%$; BPD, 51.7 vs. $14.9 \%$; a higher total number of axis I diagnoses, 4.23 (2.52) vs. 2.35 (1.76) and reported loneliness compared to clinical controls [23]. Adolescents with NSSID also had significantly more internalizing and externalizing symptoms [50]; higher levels of emotion dysregulation and general psychopathology and impairment than clinical controls $[23,50]$. The association between NSSID and clinical impairment in the study by Glenn and Klonsky [23] remained significant when controlling for BPD. An adult NSSID group also had significantly more general psychopathology and impairment [43, 45]; more symptoms of anxiety and depression [45]; more suicide attempts and ideation; were more often victims of abuse; had more previous treatment [45], ended therapy prematurely, had worse prognostic outcome after therapy than an axis I clinical comparison group but showed larger decreases on ratings of severity of illness from intake to termination as well as more improvement following therapy [43] (Table 2).

\section{NSSI disorder versus borderline personality disorder}

One study on adults distinguished potential NSSID from BPD. There were no differences in comorbidity and functional impairment between the groups. The BPD group, however, contained more women, 88 vs. $51 \%$ and reported higher rates of abuse, 54 vs. $28 \%$ [45]. The same sample was also used in a later study by Ward et al. [43], where those with NSSID showed greater improvement after treatment compared to intake than those with BPD. In one study [50] 80\% of adolescents who met NSSID criteria did not meet criteria for BPD. Glenn and Klonsky [23] found that NSSID occurred independent of BPD. There was a significant overlap between NSSID and BPD, but the diagnostic overlap between BPD and other disorders was similar to that between BPD and NSSID. Odelius and Ramklint [51] also found that patients with NSSID had several comorbid diagnoses which were not concomitant with BPD. Bracken-Minor and McDevittMurphy [54] compared BPD-positive and BPD-negative self-injuring young adults and found preliminary support for a distinction, where those with BPD reported higher levels of emotion dysregulation, 105.28 (22.95) vs. 88.31 (21.56) and functions of self-punishment, 3.90 (2.04) vs. 2.39 (2.12); anti-suicide, 2.41 (2.16) vs. 1.06 (1.87) and anti-dissociation, 2.38 (1.86) vs. 1.42 (1.73). Furthermore, the NSSI methods cutting and burning were more often reported compared to those without BPD (Table 2).

\section{Assessment of NSSI disorder}

Several studies have assessed NSSID criteria indirectly with instruments not originally developed for this purpose. The Clinician Administered Nonsuicidal SelfInjury Disorder Index (CANDI) [53] and the self-report measure The Alexian Brothers Assessment of SelfInjury (ABASI) [52] were designed to assess and identify NSSID. The CANDI showed good interrater reliability. The overall diagnostic agreement was $92 \%$. There was a $100 \%$ agreement for criteria A, B, C, D and F and $92 \%$ for criterion E. Furthermore, internal consistency was adequate and there was support for construct validity. There was support for a two-factor solution on the ABASI, with all items assessing criterion $B$ and criterion $C$ loading on respective factor. Internal consistency was adequate. Item-total correlations showed that the ABASI item for criterion B3 was weakly correlated with the NSSI severity score. Test-retest reliability was moderate for the NSSID, good for criterion $\mathrm{A}$ and criterion $\mathrm{C}$, but poor for criterion $\mathrm{B}$. Test-retest was good for ABASI NSSI severity scores and moderate for criterion $B$ and criterion $C$ subscales. In-Albon and colleagues [50] constructed a clinical interview from the DSM-5 criteria which showed very good interrater reliability. Fischer et al. [49] used a German version of the Self-Injurious Thoughts and Behaviors Interview (SITBI) [56] to identify NSSID and found moderate agreement in test-retest and very good interrater reliability. They argued that NSSI may have been triggered in their sample by the inpatient clinical setting, hence influencing test-retest results. Fischer et al. [49] suggested extending SITBI to include items on functional impairment and distress to optimally match NSSID criteria.

\section{Discussion}

Empirical data are now emerging on the DSM-5 [13] NSSID concerning prevalence rates, characteristics, proposed criteria, clinical correlates and independence from other disorders, which are important aspects when validating a new diagnosis [57]. Comparisons and conclusions are however limited by the fact that different versions of the criteria have been used and that not all criteria have been assessed or have been assessed indirectly [30]. In addition, the total number of empirical studies is still small, especially for those presenting the full final DSM-5 criteria, indicating that this is an area in need of further study. In view of the fact that limited reliability prevented the inclusion of an NSSI diagnosis in DSM-5 [40, 41], studies with psychometric data from instruments with structural assessment of NSSID $[52,53]$ have shown promising results.

\section{NSSI disorder criteria}

Since NSSI has shown to be a common phenomenon in adolescents, both in clinical and community samples $[2,3]$, it is important to differentiate between those who 
engage in the behavior once or twice and those who do so more repetitively. In a sample of young adolescents with high endorsement of NSSI, for example, Bjärehed et al. [58] found that a high proportion of adolescents only reported low levels of frequent NSSI and also low levels of associated psychological problems. Previous research has shown support for a distinction between occasional and repetitive NSSI, with frequent NSSI being associated with more psychopathology $[14,58]$. In several studies five instances has come to represent repetitive NSSI $[14,58]$. With regard to the DSM-5 [13] cut-off of five instances, a study by Zetterqvist et al. [46] showed that a majority of adolescents in a community sample reported engaging in NSSI more than 11 times during the past year. In clinical child and adolescent psychiatry practice, adolescents often report far higher frequencies, giving the impression that five is perhaps a low limit for adolescents. This is thus an area that needs looking into in more detail. Furthermore, as criterion A is currently stated, no significance is given to potential differences between severe and minor NSSI methods in relation to the number of instances, and this also needs some further elaboration [30]. Some of the self-report measures used to operationalize NSSI criteria include NSSI methods where there might be uncertainty whether they induce actual bleeding, bruising or pain. As Washburn and colleagues [52] pointed out, this might result in an overestimation of criterion A. To address this, some studies have excluded some methods so as to arrive at conservative estimates [46, 52]. Most participants with NSSID, however, endorsed several different NSSI methods, which might reduce this risk. That NSSI was preceded by negative feelings or relational difficulties (C1) and relieved negative states (B1) were commonly endorsed criteria $[23,46$, 50, 52]. Lengel and Mullins-Sweatt [42] also found that these features were assessed by many clinicians as prototypic symptoms of the NSSID diagnosis. Criteria B2, B3 and C2, C3 were relatively less frequently endorsed. Specifically, experiencing negative emotions prior to NSSI was highly endorsed, confirming the motivation for affect regulation as a central aspect of the NSSID construct. There was a clear difference between adults and adolescents in the endorsement of criterion B2 (resolving an interpersonal difficulty). This is in line with previous research showing that interpersonal functions are more common in adolescents than in adults [59, 60]. In one adolescent sample [52], criterion B3 (inducing positive feeling) was least commonly endorsed, and there is an ongoing discussion of the positive and negative aspect of the automatic reinforcement of NSSI [61-63]. Based on their results, Washburn and colleagues [52] raised the issue that perhaps criterion $B$ is superfluous in relation to criterion $\mathrm{C}$ and that a combination of the two would result in more parsimonious criteria. In one study of adults, over $10 \%$ responded "I don't know" to criterion $B$ items [55]. Perhaps precipitating events are easier to consciously observe than consequences of behaviors. This could also imply that the wording of the B criterion needs to be clarified for a more precise definition. Can B3 also refer to pain, stimulation and satisfaction [62]? Selby et al. [30] have also pointed out that the B3 criterion could preferably be expanded to include feeling generation/anti-dissociation when feeling numb or empty [46, $59,61,62]$.

One potential explanation why more girls than boys meet NSSID criteria is perhaps that boys traditionally are less inclined to acknowledge the emotional and motivational aspects of the diagnosis [46, 47]. Interpretations of gender differences should, however, be made with caution since there was female overrepresentation in samples. Several of the empirical studies in this review have drawn attention to the fact that criterion $\mathrm{E}$ received a relatively lower endorsement. That NSSI tends to be regarded as a solution, reducing distress rather than causing it, has previously been problematized by Wilkinson and Goodyer [33] with regard to the wording of criterion E. Clinicians also rated criterion $\mathrm{E}$ as less prototypic, suggesting that while clinicians were concerned with NSSI and its consequences, individuals with NSSI may not always perceive themselves as impaired in their everyday lives [42]. It is somewhat problematic that different operationalizations of criterion $\mathrm{E}$ have been used in the empirical studies of NSSID. Some, for example, have assumed impairment based on the fact that participants are in psychiatric inpatient clinics, while others have asked if participants wanted help for their NSSI. Compared to other diagnoses, such as ADHD or depression, where the distress/ impairment criterion is more easily applied, it is perhaps necessary with further instructions how this criterion should be operationalized so as not to exclude individuals incorrectly. Gratz et al. [53] showed that criterion E best distinguished NSSID from those with NSSI not meeting criteria for the disorder, which implies that it is important for the validity of the construct and, as such, potentially functions appropriately by screening out those without distressing or impairing NSSI.

\section{NSSI disorder as a separate diagnostic entity}

Using the DSM-5 criteria [13], a sample of individuals was identified who had more general psychopathology and impairment than both clinical controls and those with NSSI not meeting criteria for NSSID, preliminarily supporting that NSSID can be reliably identified among selfinjurers. Importantly, the differences remained significant after BPD was controlled for $[23,53]$ and NSSID was preliminary found to be distinguishable from BPD [50,54]. In 
adolescents, for example, each disorder explained unique variance in emotion regulation deficits [23]. Furthermore, BPD-positive self-injurers with NSSID reported higher levels of emotion dysregulation than BPD-negative selfinjurers with NSSID [54]. Support for the independence of NSSID should be based on an overlap between NSSID and BPD to the same extent as other disorders, as pointed out by Glenn and Klonsky [23]. Similarly, suicidal behaviors also co-occur with depression, PTSD, substance abuse and eating disorders, for example, as well as several other clinical behaviors and thus an overlap between NSSI and suicidal behaviors is not necessarily evidence per se against a distinction between the two.

\section{Future work}

Future work in the research field of NSSI would benefit from a unified conceptualization of NSSI with standardized assessment measures in order to facilitate comparisons and achieve more consistent results. The proposed NSSID diagnostic criteria [13] are a step towards a mutually agreed-upon conceptualization [3]. Although most criteria were possible to apply and were assessed as prototypical, some clarification of criteria is perhaps needed in order to facilitate clinical assessment. Future studies are needed to assess whether all suggested criteria are equally meaningful clinically. The prevalence rates of the final DSM-5 [13] NSSID criteria need to be further verified in both clinical and community groups of adolescents by other methods than self-report, such as diagnostic interviews, to further assess reliability and validity of a potential NSSID diagnosis. It is also important to collect more data on male samples. Further studies on overlapping and unique correlates to NSSID are also needed, as are longitudinal studies in order to examine risk factors and the prognosis of NSSID, and its relationship to diagnostic neighbors and suicidal behaviors over time.

\section{Conclusion}

When the DSM-5 NSSID criteria were used in the reviewed empirical studies, a group of adolescents and young adults was identified that was clinically more severe in comparison both with those with NSSI not meeting NSSID criteria and with clinical controls. There was also preliminary support for the independence of NSSID and a distinction in relation to BPD. In order to accumulate data to validate and reliably assess a potential NSSID, further empirical studies are needed using the full and final DSM-5 [13] criteria.

\footnotetext{
Author details

${ }^{1}$ Department of Clinical and Experimental Medicine, Linköping University, 58185 Linköping, Sweden. ${ }^{2}$ Department of Child and Adolescent Psychiatry, Linköping University, 58185 Linköping, Sweden.
}

\section{Compliance with ethical guidelines}

\section{Competing interests}

The author declares that she has no competing interests.

Received: 23 March 2015 Accepted: 24 June 2015

Published online: 28 September 2015

\section{References}

1. International Society for the Study of Self-Injury (2007) Definition of nonsuicidal self-injury. http://www.itriples.org/isss-aboutself-i.html. Accessed 28 May 2014

2. Muehlenkamp JJ, Claes L, Havertape L, Plener PL (2012) International prevalence of non-suicidal self-injury and deliberate self-harm. Child Adolesc Psychiatry Ment Health. doi:10.1186/1753-2000-6-10

3. Swannell SV, Martin GE, Page A, Hasking P, St John MJ (2014) Prevalence of nonsuicidal self-injury in nonclinical samples: systematic review, metaanalysis and meta-regression. Suicide Life Threat Behav 44:273-303

4. DiClemente RJ, Ponton LE, Hartley D (1991) Prevalence and correlates of cutting behavior: risk for HIV transmission. J Am Acad Child Adolesc Psychiatry 30:735-739

5. American Psychiatric Association (1994) Diagnostic and statistical manual of mental disorders, 4th edn. American Psychiatric Association, Washington DC

6. Favazza AR, Rosenthal RJ (1990) Varieties of pathological self-mutilation. Behav Neurol 3:77-85

7. Favazza AR, Rosenthal RJ (1993) Diagnostic issues in self-mutilation. Hosp Community Psychiatry 44:134-140

8. Herpertz S (1995) Self-injurious behaviors. Acta Psychiatr Scand 91:57-68

9. Kahan J, Pattison EM (1984) Proposal for a distinctive diagnosis: the deliberate self-harm syndrome (DSH). Suicide Life Threat Behav 14:17-35

10. Muehlenkamp JJ (2005) Self-injurious behavior as a separate clinical syndrome. Am J Orthopsychiatr 75:324-333

11. Pattison EM, Kahan J (1983) The deliberate self-harm syndrome. Am J Psychiatry 140:867-872

12. Shaffer D, Jacobson C (2009) Proposal to the DSM-V childhood disorder and mood disorder work groups to include non-suicidal self-injury (NSSI) as a DSM-V disorder. American Psychiatric Association. http://www.dsm5. org/Pages/Default.aspx. Accessed 1 Dec 2009

13. American Psychiatric Association (2013) Diagnostic and statistical manual of mental disorders, 5th edn. American Psychiatric Association, Washington DC

14. Manca M, Presaghi F, Cerutti R (2014) Clinical specificity of acute versus chronic self-injury: measurement and evaluation of repetitive nonsuicidal self-injury. Psychiatry Res 215:111-119

15. American Psychiatric Association (2012) Suggested criteria for DSM-5 non-suicidal self-injury disorder. http://www.dsm5.org/Pages/Default. aspx. Accessed 1 May 2012

16. Andover MS, Pepper CM, Ryabchenko KA, Orrico EG, Gibb BE (2005) Self-mutilation and symptoms of depression, anxiety, and borderline personality disorder. Suicide Life Threat Behav 35:581-591

17. Glenn CR, Klonsky ED (2009) Social context during non-suicidal self-injury indicates suicide risk. Personal Individ Differ 46:25-29

18. Jacobson CM, Muehlenkamp JJ, Miller AL, Turner JB (2008) Psychiatric impairment among adolescents engaging in different types of deliberate self-harm. J Clin Child Adolesc Psychol 37:363-375

19. Klonsky ED, Oltmanns TF, Turkheimer E (2003) Deliberate self-harm in a nonclinical population: prevalence and psychological correlates. Am J Psychiatry 160:1501-1508

20. Nock MK, Joiner TE Jr, Gordon KH, Lloyd-Richardson E, Prinstein MJ (2006) Non-suicidal self-injury among adolescents: diagnostic correlates and relation to suicide attempts. Psychiatry Res 144:65-72

21. Favazza AR, Conterio K (1989) Female habitual self-mutilators. Acta Psychiatr Scand 79:283-289

22. Wilkinson P (2013) Non-suicidal self-injury. Eur Child Adolesc Psychiatry 22(Suppl 1):75-79 
23. Glenn CR, Klonsky ED (2013) Nonsuicidal self-injury disorder: an empirical investigation in adolescent psychiatric patients. J Clin Child Adolesc Psychol 42:496-507

24. Nock MK, Kessler RC (2006) Prevalence of and risk factors for suicide attempts versus suicide gestures: analysis of the national comorbidity survey. J Abnorm Psychol 115:616-623

25. Klonsky ED, May AM, Glenn CR (2013) The relationship between nonsuicidal self-injury and attempted suicide: converging evidence from four samples. J Abnorm Psychol 122:231-237

26. Klonsky ED, Muehlenkamp JJ (2007) Self-injury: a research review for the practitioner. J Clin Psychol 63:1045-1056

27. Asarnow JR, Porta G, Spirito A, Emslie G, Clarke G, Wagner KD et al (2011) Suicide attempts and nonsuicidal self-injury in the treatment of resistant depression in adolescents: findings from the TORDIA study. J Am Acad Child Adolesc Psychiatry 50:772-781

28. Wilkinson P, Kelvin R, Roberts C, Dubicka B, Goodyer I (2011) Clinical and psychosocial predictors of suicide attempts and nonsuicidal self-injury in the adolescent depression antidepressants and psychotherapy trial (ADAPT). Am J Psychiatry 168:495-501

29. Guan K, Fox KR, Prinstein MJ (2012) Nonsuicidal self-injury as a timeinvariant predictor of adolescent suicide ideation and attempts in a diverse community sample. J Consult Clin Psychol 80:842-849

30. Selby EA, Kranzler A, Fehling KB, Panza E (2015) Nonsuicidal self-injury disorder: the path to diagnostic validity and final obstacles. Clin Psychol Rev 38:79-91

31. Walsh BW (2006) Treating self-injury: a practical guide. Guilford Press, New York

32. Plener PL, Kapusta ND, Brunner R, Kaess M (2014) Non-suicidal self-injury (NSSI) and suicidal behavior disorder in the DSM-5. Z Kinder Jugendpsychiatr Psychoter 42:405-413

33. Wilkinson P, Goodyer I (2011) Non-suicidal self-injury. Eur Child Adolesc Psychiatry 20:103-108

34. Butler AM, Malone K (2013) Attempted suicide v. non-suicidal self-injury: behaviour, syndrome or diagnosis? Br J Psychiatry 202:324-325

35. Plener PL, Fegert JM (2012) Non-suicidal self-injury: State of the art perspective of a proposed new syndrome for DSM V. Child Adolesc Psychiatry Ment Health. doi:10.1186/1753-2000-6-9

36. Crowe M (2014) From expression to symptom to disorder: the psychiatric evolution of self-harm in the DSM. J Psychiatr Ment Health Nurs 21:857-858

37. De Leo D (2011) DSM-V and the future of suicidology. Crisis 32:233-239

38. Kapur N, Cooper J, O'Connor RC, Hawton K (2013) Non-suicidal self-injury v. attempted suicide: new diagnosis or false dichotomy? Br J Psychiatry 202:326-328

39. Plener PL, Kapusta ND, Kölch MG, Kaess M, Brunner R (2012) Non-suicidal self-injury as autonomous diagnosis_-implications for research and clinic of the DSM-5 proposal to establish the diagnosis of non-suicidal selfinjury in adolescents. Z Kinder Jugendpsychiatr Psychoter 40:113-120

40. Regier DA, Narrow WE, Clarke DE, Kraemer HC, Kuramoto SJ, Kuhl EA et al (2013) DSM-5 field trials in the United States and Canada, part II: test-retest reliability of selected categorical diagnoses. Am J Psychiatry 170:59-70

41. Clarke DE, Narrow WE, Regier DA, Kuramoto SJ, Kupfer DJ, Kuhl EA et al (2013) DSM-5 field trials in the United States and Canada, part I: study design, sampling strategy, implementation, and analytic approaches. Am J Psychiatry 170:43-58

42. Lengel GJ, Mullins-Sweatt SN (2013) Nonsuicidal self-injury disorder: clinician and expert ratings. Psychiatry Res 210:940-944

43. Ward A, Bender TW, Gordon KH, Nock MK, Joiner TE, Selby EA (2013) Post-therapy functional impairment as a treatment outcome measure in non-suicidal self-injury disorder using archival data. Pers Ment Health 7:69-79

44. Zetterqvist M, Lundh L-G, Svedin CG (2014) Non-suicidal self-injury in adolescents: support for a specific distress-function relationship. Child Adolesc Psychiatry Ment Health. doi:10.1186/1753-2000-8-23

45. Selby EA, Bender TW, Gordon KH, Nock MK, Joiner TE Jr (2012) Non-suicidal self-injury (NSSI) disorder: a preliminary study. Pers Disord 3:167-175

46. Zetterqvist M, Lundh L-G, Dahlström Ö, Svedin CG (2013) Prevalence and function of non-suicidal self-injury (NSSI) in a community sample of adolescents, using suggested DSM-5 criteria for a potential NSSI disorder. J Abnorm Child Psychol 41:759-773
47. Albores-Gallo L, Méndez-Santos JL, Xóchitl-Garcia Luna A, DelgadilloGonzález Y, Chávez-Flores Cl, Martínez OL (2014) Nonsuicidal self-injury in a community sample of older children and adolescents of Mexico city. Actas Esp Psiquiatr 42:159-168

48. Barrocas AL, Hankin BL, Young JF, Abela JRZ (2012) Rates of nonsuicidal self-injury in youth: age, sex, and behavioral methods in a community sample. Pediatrics 130:39-45

49. Fischer G, Ameis N, Parzer P, Plener PL, Groschwitz R, Vonderlin E et al (2014) The German version of the self-injurious thoughts and behaviors interview (SITBI-G): a tool to assess non-suicidal self-injury and suicidal behavior disorder. BMC Psychiatry 14:265

50. In-Albon T, Ruf C, Schmid M (2013) Proposed diagnostic criteria for the DSM-5 of nonsuicidal self-injury in female adolescents: diagnostic and clinical correlates. Psychiatry J. doi:10.1155/2013/159208

51. Odelius CB, Ramklint M (2014) Clinical utility of proposed non-suicidal self-injury diagnosis - a pilot study. Nord J Psychiatry 68:66-71

52. Washburn JJ, Potthoff LM, Juzwin KR, Styer DM (2014) Assessing DSM-5 nonsuicidal self-injury disorder in a clinical sample. Psychol Assess. doi:10.1037/pas0000021

53. Gratz KL, Dixon-Gordon KL, Chapman AL, Tull MT (2015) Diagnosis and characterization of DSM-5 nonsuicidal self-injury disorder using the clinician-administered nonsuicidal self-injury disorder index. Assessment. doi:10.1177/1073191114565878

54. Bracken-Minor KL, McDevitt-Murphy ME (2014) Differences in features of non-suicidal self-injury according to borderline personality disorder screening status. Arch Suicide Res 18:88-103

55. Andover MS (2014) Non-suicidal self-injury disorder in a community sample of adults. Psychiatry Res 219:305-310

56. Nock MK, Holmberg EB, Photos VI, Michel BD (2007) Self-injurious thoughts and behaviors interview: development, reliability, and validity in an adolescent sample. Psychol Assess 19:309-317

57. Robins E, Guze SB (1970) Establishment of diagnostic validity in psychiatric illness: its application to schizophrenia. Am J Psychiatry 126:983-987

58. Bjärehed J, Wångby-Lundh M, Lundh L-G (2012) Nonsuicidal self-injury in a community sample of adolescents: subgroups, stability, and associations with psychological difficulties. J Res Adolesc 22:678-693

59. Lloyd-Richardson EE, Perrine N, Dierker L, Kelley ML (2007) Characteristics and functions of non-suicidal self-injury in a community sample of adolescents. Psychol Med 37:1183-1192

60. Klonsky ED (2007) The functions of deliberate self-injury: a review of the evidence. Clin Psychol Rev 27:226-239

61. Dahlström Ö, Zetterqvist M, Lundh L-G, Svedin CG (2015) Functions of nonsuicidal self-injury: exploratory and confirmatory factor analyses in a large community sample of adolescents. Psychol Assess 27:302-313

62. Selby EA, Nock MK, Kranzler A (2014) How does self-injury feel? Examining automatic positive reinforcement in adolescent self-injurers with experience sampling. Psychiatry Res 215:417-423

63. Klonsky ED (2009) The functions of self-injury in young adults who cut themselves: clarifying the evidence for affect-regulation. Psychiatry Res 166:260-268

\section{Submit your next manuscript to BioMed Central and take full advantage of:}

- Convenient online submission

- Thorough peer review

- No space constraints or color figure charges

- Immediate publication on acceptance

- Inclusion in PubMed, CAS, Scopus and Google Scholar

- Research which is freely available for redistribution

Submit your manuscript at

www.biomedcentral.com/submit
() Biomed Central 